\title{
Genetically Modified Striatal Astrocytes Grafted to the Brain: Pharmacological Control of an Inducible Promoter
}

\author{
Edmund F. La Gamma ${ }^{1}$, Gary Weisinger ${ }^{1}$, Nicholas J. Lenn ${ }^{2}$ and Robert E. Strecker ${ }^{3}$ \\ Departments of Pediatrics \& Neurobiology ${ }^{1}$, Neurology $^{2}$, Psychiatry $^{3}$, \\ SUNY at Stony Brook, NY 11794, USA
}

In the late 1980s, several investigators applied the strategy of combining genetic engineering with brain grafting (for review see Gage et al., Neuron 1991; 6: 1). Disadvantages associated with current approaches include the use of nonbrain donor tissue (fibroblasts), the use of tumor-derived cell lines, and the use of viral infection methods to introduce the gene of interest. We report here the use of the non-viral, calcium phosphate transfection method to introduce stably expressed genes into primary cultures of striatal astrocytes for subsequent use in brain grafting. Astrocytes may be the preferred cellular vehicle for gene replacement therapy in brain transplantation paradigms because they divide in culture, are brain-derived and regionally specific, possess a release mechanism, secrete growth factors, can migrate several millimeters from the transplant site, and have neurotransmitter receptors. In addition, we introduce and demonstrate the regulation of gene product expression by systemically administered drugs. Thus, by the incorporation of inducible promoters in the transfected gene sequence, drugs which act upon astrocyte neurotransmitter receptors can regulate the level of transfected gene expression.

Neonatal (day 2) rat striatal astrocytes were transfected with RSV-driven or enkephalin promoter-driven chloramphenicol acetyl transferase (CAT) chimeric plasmids after 3 weeks in culture. In addition, the incorporation of the neomycin resistance gene combined with selective growth of transfected cells in G418containing ( $300 \mu \mathrm{g} / \mathrm{ml})$ media for 3 additional weeks insured stable transfected gene incorporation from all surviving astrocytes. These cultured astrocytes expressed CAT enzymatic activity, which was measured both in cell lysates and in the conditioned media. As the cells in these cultures were $100 \%$ viable, the latter observation indicates that the astrocytes secreted CAT into the media. Following transplantation into the adult rat striatum, the transplanted astrocytes were identified histologically by astrocyte-specific GFAP staining, as well as with antibody staining to CAT protein to specifically detect the transfected cells. To be certain that GFAP and CAT staining identified transplanted cells, CAT activity was also biochemically detected in tissue blocks containing the transplant at both 1 day and 3 weeks after transplantation.

Pharmacological regulation of gene product expression was demonstrated using transfected astrocytes containing the human preproenkephalin (ppEnk) promoter in the gene sequence. Regulation of the ppEnk gene by cAMP-dependent second messenger mechanisms has been studied previously (Comb et al., Nature 1986; 323: 353). We studied in vitro the effect of various dopaminergic and serotonergic agonists, both of which can act via cAMP pathways in astrocytes (Hansson, Prog Neurobiol 1988; 30: 369). Serotonin agonists did not affect the expression of the transfected gene in striatal astrocytes. However, dopaminergic receptor agonists (dopamine, apomorphine, SKF38393, quinpirole) produced increases in transfected CAT gene expression in vitro as much as 20 -fold (see Figure, from Weisinger et al., submitted). This effect was blocked by dopamine antagonists. Regulation of the inducible enkephalin promoter in astrocyte transplants in vivo has also been successful. Amphetamine ( $3 \mathrm{mg} / \mathrm{kg}$, i.p., T.I.D.) produced approximately a 2 -fold increase in graft-derived CAT activity. Conversely, grafts placed in dopamine denervated striata con- 


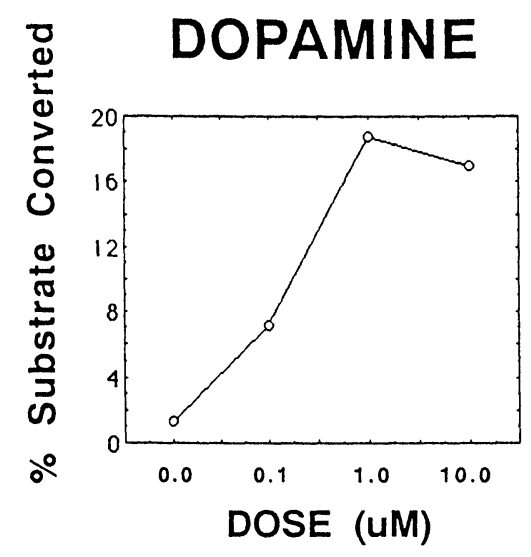

tained $30 \%$ less CAT activity than did grafts in the intact/contralateral striata. Gene regulation by drugs would enable transplant protocols to control the regulation of any transfected gene

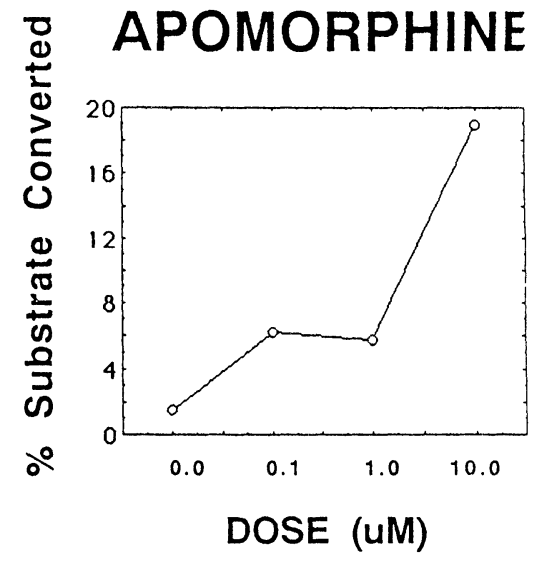

product (e.g., growth factors, transmitters, receptors, etc.) on command during development or after brain injury.

Supported in part by NIH Grant No. RR05736. 

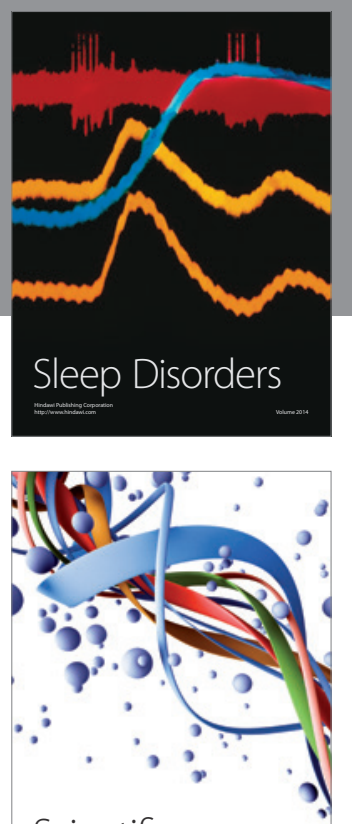

Scientifica
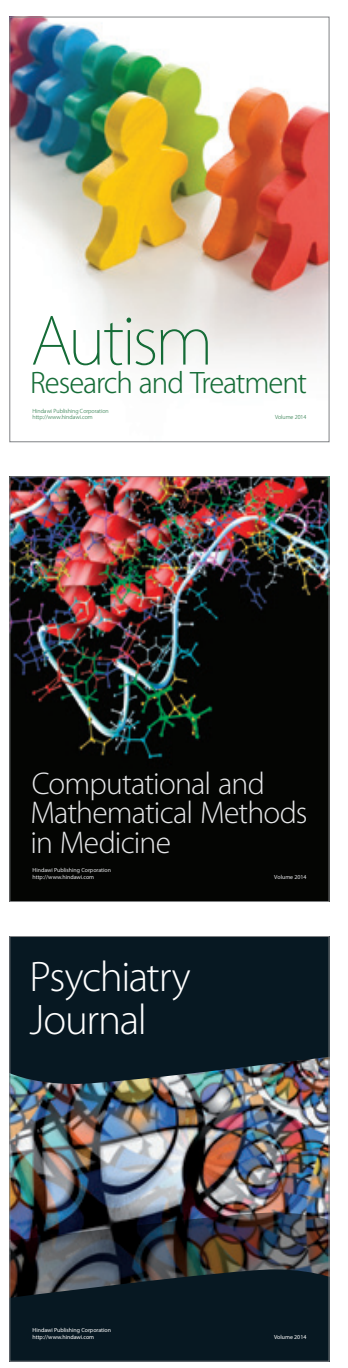
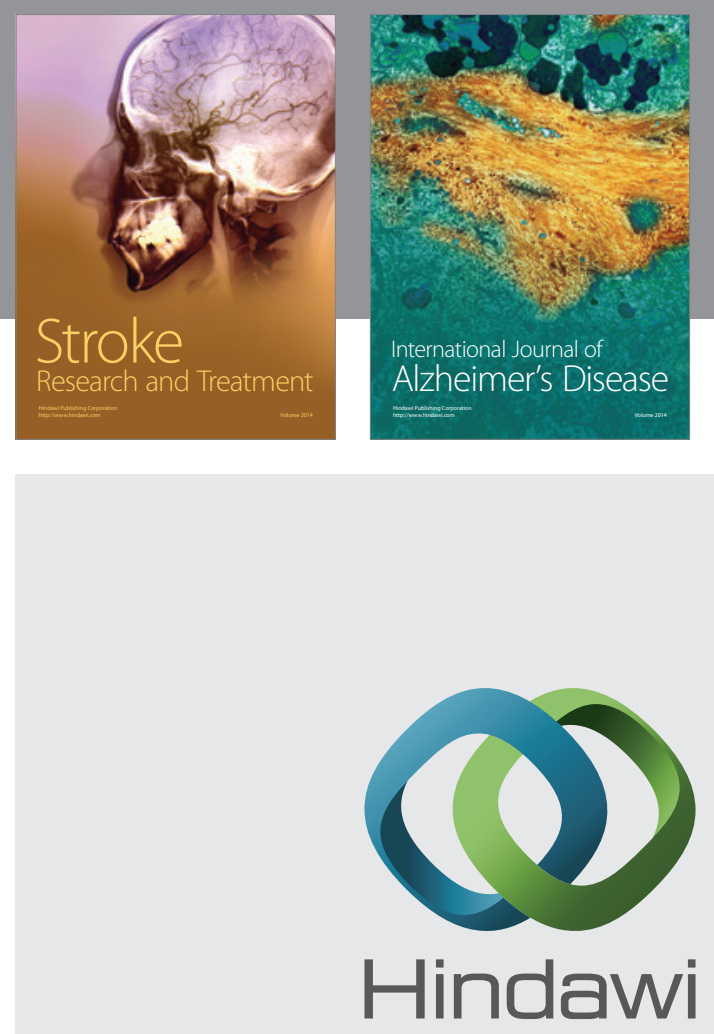

Submit your manuscripts at

http://www.hindawi.com
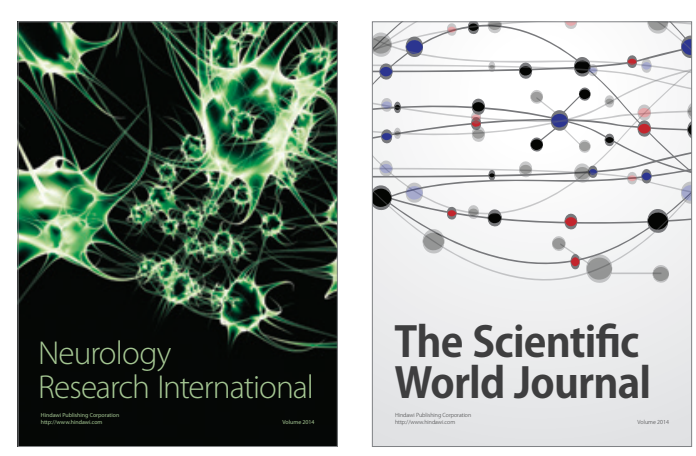

The Scientific World Journal

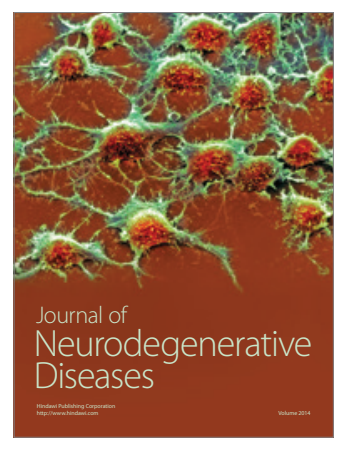

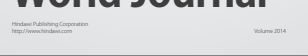

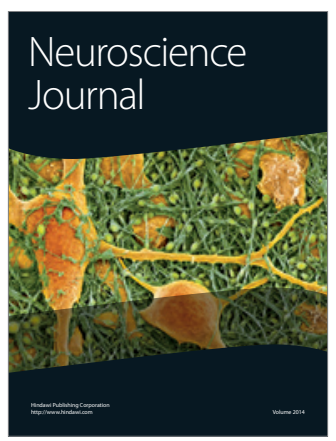

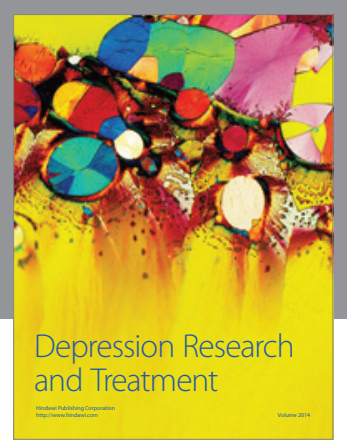
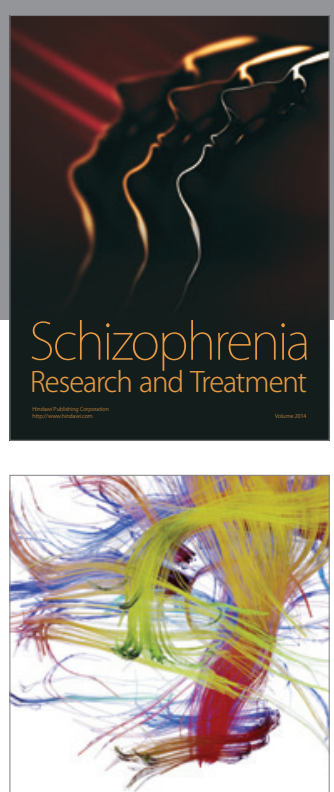

Brain Science

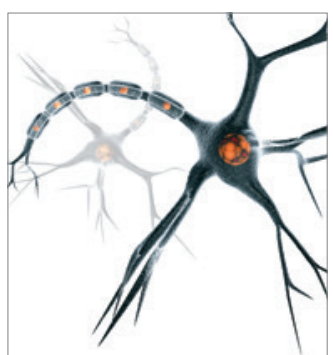

Neural Plasticity
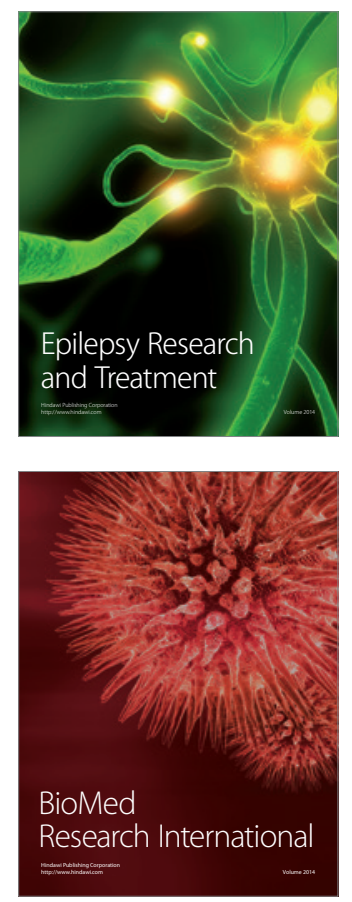

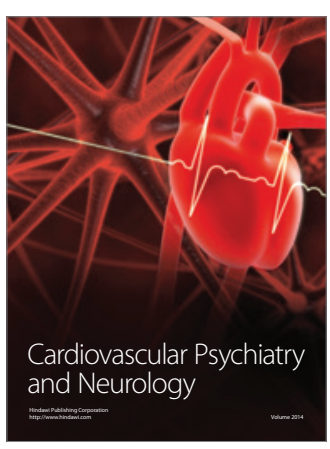

Parkinson's

Disease
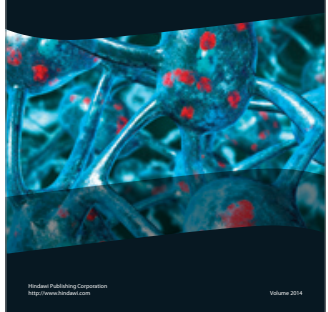